\title{
Pierre Gisel, (éd.), Les constellations du croire. Dispositifs hérités, problématisations, destin contemporain
}

Genève, Labor et Fides, 2009, 201 p.

Daniel Vidal

\section{(2) OpenEdition Journals}

Édition électronique

URL : http://journals.openedition.org/assr/21573

DOI : $10.4000 /$ assr. 21573

ISSN : $1777-5825$

Éditeur

Éditions de l'EHESS

Édition imprimée

Date de publication : 31 décembre 2009

ISBN : 978-2-7132-2218-4

ISSN : 0335-5985

Référence électronique

Daniel Vidal, «Pierre Gisel, (éd.), Les constellations du croire. Dispositifs hérités, problématisations, destin contemporain », Archives de sciences sociales des religions [En ligne], 148 | octobre-décembre 2009, document 148-58, mis en ligne le 15 novembre 2012, consulté le 21 septembre 2020. URL : http://journals.openedition.org/assr/21573 ; DOI : https://doi.org/10.4000/assr.21573

Ce document a été généré automatiquement le 21 septembre 2020.

(c) Archives de sciences sociales des religions 


\title{
Pierre Gisel, (éd.), Les constellations du croire. Dispositifs hérités, problématisations, destin contemporain
}

\author{
Genève, Labor et Fides, 2009, 201 p.
}

Daniel Vidal

\section{RÉFÉRENCE}

Pierre GISEL, (éd.), Les constellations du croire.

Dispositifs hérités, problématisations, destin contemporain, Genève, Labor et Fides, 2009, $201 \mathrm{p}$.

148-58

Croire est toujours un acte paradoxal : il énonce quelque chose au-delà ou en dehors du savoir, en un registre entièrement différent de ce qui gouverne la raison de science mais qui fonde sa légitimité, et son urgence, sur quelque " rationalité » déployée, nous le verrons, du centre même de la subjectivité du « croyant ». S'interroger dès lors sur le «croire » et ses modalités, ses « constellations », les conditions de sa permanence dans la modernité occidentale, c'est s'engager au plus profond de ce paradoxe, en identifier les apories mais aussi les possibilités de dépassements, en définir les seuils et leurs transgressions. Les différentes contributions regroupées par Pierre Gisel témoignent à la fois de cette vertigineuse descente au cœur de la subjectivité, où l'on pourrait penser que le « croire » s'exténue, en même temps qu'elles attestent la magnification du croire comme moment capital d'une modernité «en l'absence de Dieu, " et qui serait à interpréter comme l'accomplissement même, et pour ainsi dire la perfection, du christianisme. Dès l'ouverture de l'ouvrage, et dans l'examen de la thématique et des différentes «facettes» du croire, Pierre Gisel définit l'acte de croire comme question 
centrale en "occident ", - qui me paraît être, sous sa plume, ainsi que chez d'autres auteurs, métaphore obligée de la modernité - car s'y joue une véritable «dramatique de l'âme » : l'autonomie du sujet suppose dans le même temps, l'appel à transcendance, d'autant plus impératif que le croire est acte pleinement assumé d'un sujet conscient. Il n'est de subjectivité que dans une relation à une altérité radicale, qui donne sens à la finitude du sujet. Le "croire » est cette relation. Elle s'établit entre un sujet situé au sein d'une socialité - ensemble de "pratiques et d'institutions de sens»-, et le "dépassement» de toute fonctionnalité et de tout appel à salut ou à gratification. La foi, qui, pour la plupart des auteurs, n'est que l'autre nom du croire, relève donc d'abord de "l'existence en acte", en tant que cette existence est "suspendue à un achèvement d'un autre ordre ». Et tel croire « inscrit au cœur du sujet», en sa qualité d'existant, de sujet assumant sa subjectivité, échappe à la question de la vérité et de la fausseté. Aussi bien ne serait-il pas pertinent de lui opposer les critères de «raison ", puisque cette vocation à transcendance est humaine, trop humaine.

2 Il ne suffit pas de dire que le "religieux» marque un écart "par rapport aux généralités et contraintes du réel » : il est à la fois cette marque et le nom de cet écart, ce signe et cet abîme. Croire est à la fois désigner l'écart, le manque, et habiter ce manque comme sa raison. Luc-Thomas Somme rappellera dans son intervention Hugues de Saint-Victor, qui conjoint savoir et manque, science et catastrophe du réel: «La foi est une certitude de l'esprit, portant sur des réalités absentes ». Si l'on peut admettre, sous réserve que chaque glissement soit bien contrôlé, la transition sémantique entre « croire », « foi », « religieux », il est plus surprenant, à ce moment de l'analyse, que nulle référence ne soit faite à l'œuvre de Kierkegaard. Il est vrai que P. Gisel n'est pas sans en solliciter, mais à voix basse, l'expérience spirituelle, quand il signale le «croire» existentiel soumis à la rude épreuve du doute ( il est exposé à démenti et à correction»), et que, "faillible, toute croyance est particulière et contingente ». D'être tel, le "croire » définit l'espace d'une subjectivité traversée de culpabilité et de tremblement. Il ne s'agit cependant pas, ici, d'une réponse à une injonction venue d'ailleurs : mais d'un retournement de la subjectivité sur elle-même. Le « croire » est ce « travail spirituel » qui ne cesse de faire épreuve contre soi-même à partir de soi.

3 Si l'ouvrage pose avec insistance le croire comme armature de la subjectivité, il veut en donner définition «à même le monde et en fonction du monde ». Un couplage se fonde ainsi entre l'espace du sujet et l'espace du "monde », hors de tout désengagement. L'âge moderne invite en effet à cette réinscription du croire dans l'appartenance au monde "réel », en son historicité et sa temporalité. Et cette histoire est composite, plurielle, sans cesse enjoignant au croire de se réaménager, de se recomposer, au rythme des marées du temps. Mais il semble intervenir en ce point une difficulté. P. Gisel paraît enclore le croire dans sa racine comptable: croyance-créance; foifinance; fidéisme-fiduciaire ; croire-crédit: "le croire est un faire-crédit sur fond d'existence collective, qui comprend ou ressortit à l'échange, à un do ut des, voire à un sacrifice ». Ne glisse-t-on pas alors d'un croire comme attestation nue d'un manque (que ce manque se dise « mort de Dieu » ou inconnaissance d'un ordre référentiel - d'où le «doute " et la "culpabilité » sur soi retournée), à un croire comme motion (émotion ?) vers une transcendance malgré tout toujours déjà là en attente de retour ? Le croire serait-il marchandise, quand il paraissait être pur don, tout retour, précisément, aboli ? Le croire comme conjuration de la perte, ou le croire comme cette 
perte même? P.Gisel dit très justement que le croire est affaire intime d'excès, toujours lié "à ce qui dépasse la maîtrise et l'appropriable », et que, dans le champ institutionnel, s'ensuivent rites et mythes capables de canaliser cet excès vers des accomplissements sociétaux. Mais l'excès relève toujours d'une subjectivité portée au plus haut degré de sa tension, lorsque, dans "l'espace de l'intériorité », l'appel à " transcendance » s'énonce comme la résolution d'un conflit entre une conscience de la finitude et la conscience d'un manque essentiel. Le croire est alors le nom de cette résolution. Tout entier reversé au compte de cette instance conflictuelle de la subjectivité, le croire peut se définir comme "circonscription du religieux»: il demeure dans le plein pouvoir du sujet. La transcendance est le sujet en tant que toujours dédoublé.

4 Il est important, en cette phase de l'analyse, que L.T. Somme rappelle l'argument de Thomas d'Aquin, qui, au-delà de l'opinion et en deçà de la science, définit le "croire " comme articulation dynamique entre intelligence et volonté, entre acte de recherche et délibération. «Nul ne croit s'il ne le veut ». Certes, le «credere » est un acte « intérieur à la foi », qui donc présuppose cette «foi» comme sa condition. Mais cet « assentiment » primordial est affaire de choix existentiel - et ne décide en rien d'un « objet » au-delà. Le " croire » peut alors se dire « intrinsèquement volontaire ", c'est-àdire ressortissant de la seule compétence du sujet, de son seul désir. Et il n'est en effet de désir que fondé sur l'absence. Croire est alors la modalité la plus scandaleuse du pari pascalien. Le lecteur peut s'étonner que nulle référence ici ne soit faite à l'auteur des Pensées... Il est vrai que la thèse est contenue en germe, mais selon un mode discursif différent, chez Thomas d'Aquin : «L'imperfection de la connaissance est essentielle à la foi. Elle est dans sa définition ». En cette « imperfection », il convient de lire infiniment plus qu'un échec du savoir : une "nuit totale » écrit Pessoa - un manque fondateur, en quoi tout désir et tout "croire » trouvent leur origine. De ce "manque » viennent «l'inquiétude et l'insatisfaction du croyant ». Telle est la dure loi du croire.

Le « croire » est excès, « dépassement, élargissement, dépossession » - à l'image, si l'on peut dire, de Dieu, qui est cet excès aussi bien, qu'évoquent les grands poètes mystiques $\mathrm{du} \mathrm{XVII}^{\mathrm{e}}$. L'existence est ainsi, selon une formule désormais banale, cette "sortie de soi ». La croyance est cette " excentricité de la foi » que Luther "pousse à l'extrême ", selon la réflexion de Hans-Christoph Askani. En divergence avec l'argument luthérien, l'auteur rappelle la position de Paul Tillich, qui définit le croire, la foi, comme « courage d'être » de l'homme en tant que « fini » participant à «l'infini dans son courage, dans la transcendance de ses préoccupations finies vers une préoccupation infinie, absolue, ultime ». C'est parce qu'il est exposé à sa finitude, que l'être humain, «à la force du non-être, sait répondre grâce à la force de l'être-même (...) l'homme ose être ». Et ce " courage d'être", sur fond "de l'absurde, au sein du désespoir ", fonde la relation " entre l'être humain et l'Être ", et par là l'union entre Dieu et l'homme, garantie par la doctrine d'une justification venue de "Dieu seul ». L'angoisse est ainsi au principe de la «rencontre » et de la "participation» de l'homme à son dieu. Au contraire, ou au revers, de la théologie de l'être, Luther formule une théologie de la Parole comme jugement, et donc porteuse d'exclusion. La parole est seule, - la seule -, ou elle n'est pas. Elle introduit une distance incommensurable entre le croyant et Dieu, dans la mesure où croire est être saisi dans les filets de la parole, dans sa "gratuité », son «instabilité », son «infini ». Et que cette saisie est nécessairement impossible, impensable : croire est ainsi en permanence flotter dans l'univers "suspendu » de la Parole, de laquelle rien ne peut se "déduire », puisqu'elle est à elle-même sa propre 
résolution. Croire est aussitôt entrer dans l'univers morbide, et le seul cependant habitable, de la culpabilité. La « foi seule » est attestation de cette science et conscience de la Faute.

6 Il se peut que le "croire post-moderne ", qu'analyse Carlos Mendoza, ne soit pas si éloigné de cette conception. D'une part en ce qu'il échappe à tout absolu, le sujet du croire étant de «condition pronominale relationnelle, action performative ambiguë, créatrice de sens toujours provisoire ». Par-delà Tillich, clin d'œil de Derrida à Luther ? Non, sans doute. Mais la réhabilitation de l'intentionnalité du sujet connaissant, dans sa «faiblesse » même, dans sa précarité, sa pauvreté, sa vulnérabilité, plaide pour une interprétation de la «foi » et du «croire » comme « événements » toujours singuliers, actes d'une subjectivité qui s'institue d'une "irruption de l'Autre» toujours particulière. Le "mystère de Dieu ", et sa profondeur, notait Askoni à propos de Luther, " consiste en ce que le mystère de l'homme n'est pas moins profond ». Le "croire " postmoderne écarte ces notions de mystère mais en réintroduit la tension en dépossédant patiemment le sujet de ses cadrages et raisons. Face à un monde « inappropriable » (Thierry Laus), et dans le désir d'échapper à la mort (Christian Indermuhle), le croire est réponse aux plus hauts défis de l'être : il ne peut simplement faire « comme si » ce monde en amont était de texture intelligible, sans pour autant le tenir pour point d'absolu aveuglement. L'acte de croyance ne peut non plus faire l'économie d'une pensée de la mort, d'une pesée franche et sans simulacre de cette mort au bout. Car la mort est "le seuil délimiteur à partir duquel se dessine la possibilité du langage » - et, avec le langage, la possibilité de franchir les frontières du sens et de son monde. Un après-coup demeure alors toujours pensable, et dicible. Quelque soit le mode de rapatriement, dans la subjectivité, de cette pensée et de ce pas au-delà. Dieu, l'éternité, le salut, etc. sont signifiants d'ici et maintenant, et de pure présence. Le « croire » est attestation de cette présence.

7 Interrogeant la «constellation » contemporaine du croire, et la revendication d'une "religiosité sans religion », Serge Mangel précise que le croire, loin de témoigner d'une déperdition du religieux, au profit d'une croyance désacralisée, s'inscrit à l'inverse au centre d'un dispositif déjà là: "une chrétienté sans religion chrétienne, sans institution publique... Une chrétienté déjà là, inscrite dans la raison naturelle ». Il semble ainsi que se boucle le parcours du croire. À condition de créditer, si l'on peut dire, la chrétienté, de cette "universalité de la raison devenue consciente d'ellemême », et de définir les critères de cette "nouvelle topographie de la croyance ». Des Évangiles viennent les prescriptions morales qui ont valeur de loi, au-delà de la foi et du savoir. Plus exactement, et dans la suite de Kant, S. Mangel privilégie la «foi réfléchissante ", par quoi « le sujet se donne à lui-même les principes d'universalité de la Loi », et fonde ainsi l'acte de croyance en la rationalité de la loi morale. Il s'ensuit, note l'auteur, qu'« avec le christianisme, la raison n'a plus besoin de Dieu pour croire en Dieu » - ou pour affecter d'un coefficient de croyance, quelque signifiant majeur d'altérité. La référence à Freud et au "meurtre du père " comme "équivalent symbolique » de la «mort de Dieu » est ici très précieuse. Car la « raison » n'intervient pas comme clôture et plénitude du concept. Ressortissant à l'ordre du «fini », la raison doit n'être que pure abstraction pour s'ouvrir à la recherche «des idées transcendantales ». Alors prend sens la thèse kantienne d'une "croyance issue directement de la rationalité elle-même ". Quand le judaïsme nie, à travers le fils, le meurtre du Père, le christianisme en fait le plein aveu. C'est au prix de cet aveu que le christianisme peut fonder un croire en raison morale, cette raison en abstraction de la 
loi, et cette abstraction en "fondement ultime de la subjectivité ». Si "croire, en modernité, est avouer la mort de Dieu », un bouleversement radical intervient, qui rend pour l'essentiel caduque la séparation convenue du savoir et de la croyance, de soimême en tant que soi et de soi-même comme un autre. Une neuve conception du sujet et $\mathrm{du}$ monde réel devrait désormais pouvoir s'énoncer, au-delà du christianisme, comme au-delà du mal et du bien. Le croire, comme acte de présence au seul monde. Ici et maintenant. 